\title{
Pharmacology and Surface Electrostatics of the K Channel Outer Pore Vestibule
}

\author{
Claire C. Quinn, Ted Begenisich \\ Department of Pharmacology and Physiology, University of Rochester Medical Center, 601 Elmwood Avenue, Rochester, NY 14642, USA
}

Received: 6 June 2006/Revised: 6 September 2006

\begin{abstract}
In spite of a generally well-conserved outer vestibule and pore structure, there is considerable diversity in the pharmacology of $\mathrm{K}$ channels. We have investigated the role of specific outer vestibule charged residues in the pharmacology of $\mathrm{K}$ channels using tetraethylammonium (TEA) and a trivalent TEA analog, gallamine. Similar to Shaker K channels, gallamine block of Kv3.1 channels was more sensitive to solution ionic strength than was TEA block, a result consistent with a contribution from an electrostatic potential near the blocking site. In contrast, TEA block of another type of $\mathrm{K}$ channel (Kv2.1) was insensitive to solution ionic strength and these channels were resistant to block by gallamine. Neutralizing either of two lysine residues in the outer vestibule of these $\mathrm{Kv} 2.1$ channels conferred ionic strength sensitivity to TEA block. Kv2.1 channels with both lysines neutralized were sensitive to block by gallamine, and the ionic strength dependence of this block was greater than that for TEA. These results demonstrate that Kv3.1 (like Shaker) channels contain negatively charged residues in the outer vestibule of the pore that influence quaternary ammonium pharmacology. The presence of specific lysine residues in wild-type Kv2.1 channels produces an outer vestibule with little or no net charge, with important consequences for quaternary ammonium block. Neutralizing these key lysines results in a negatively charged vestibule with pharmacological properties approaching those of other types of $\mathrm{K}$ channels.
\end{abstract}

Claire Quinn's current address is: Department of Pharmacology, Apuit Limited, Research Avenue South, Heriot Watt University Research Park, Riccarton, Edinburgh, EH14 4AP, United Kingdom.

Correspondence to: Ted Begenisich; email: ted_begenisich@ urmc.rochester.edu
Key words: $K$ channel - Surface potential Quaternary ammonium ion - Channel block Tetraethylammonium — Gallamine

\section{Introduction}

More than 80 mammalian genes code for $\mathrm{K}$ channel protein subunits, so it is not surprising that the physiological roles of $\mathrm{K}$ channels include such diverse actions as controlling action potential duration, action potential firing patterns and salt and water movement (Hille, 2001). Given the important, complex physiological processes controlled by $\mathrm{K}$ channels, it is not surprising that considerable effort has been expended on understanding their pharmacological properties (Tamargo et al., 2004; Yost, 1999). K channels are blocked by many chemical agents, including many types of complex peptides, especially peptide toxins, and by simple amine compounds, including the quaternary amine tetraethyl ammonium (TEA).

The study of the pharmacology of $\mathrm{K}$ channels has been greatly aided by mutational analysis of cloned $\mathrm{K}$ channels and the solution of the crystal structures of several bacterial $\mathrm{K}$ channels (Doyle et al., 1998; Jiang et al., 2002, 2003) and a mammalian voltage-gated flavor (Long, Campbell \& MacKinnon, 2005). This work has revealed a remarkable conservation of the general structure of the outer vestibule and selectivity filter of $\mathrm{K}$ channels. In spite of this structural conservation, there are considerable differences in the pharmacological properties of $\mathrm{K}$ channels.

Compared to the peptide toxins and other agents that inhibit $\mathrm{K}$ channels, the simple quaternary amine TEA ought to be the easiest to understand. Early mutational analysis revealed a "hot spot" for external TEA block: the affinity for this compound changes over more than two orders of magnitude depending on the amino acid at this position (Kavanaugh et al., 
1991; MacKinnon \& Yellen, 1990). Additional studies revealed that an aromatic amino acid side chain at this hot spot on all four subunits of the homotetrameric channels contributes to high-affinity TEA binding (Heginbotham \& MacKinnon, 1992; Kavanaugh et al., 1992). This amino acid (position 449 of the prototypical voltage-gated K channel, Shaker) is located just outside the entrance to the pore selectivity filter, and all the data appeared consistent with the idea that TEA was stabilized in its blocking site through cation $\pi$-electron interaction with this aromatic side chain.

More recent studies, however, have suggested that the mechanism of TEA and other quaternary ion block of K channels is more complex. Analysis of the structure-activity relations for $\mathrm{K}$ channel block of quaternary amines revealed contributions from hydrophobic interactions as well as a role for dehydration of the blocking ions (Jarolimek et al., 1995, 1996). In addition, amino acids other than that at position 449 are involved in TEA binding (Pascual et al., 1995). A molecular dynamics study (Crouzy, Berneche \& Roux, 2001) showed that K channel proteins do not have the three-dimensional geometry necessary for TEA: $\pi$-electron interaction. Finally, studies of the ability of TEA to alter the chemical modification of introduced cysteine residues indicate that TEA likely blocks at a location more external than position 449 (Andalib et al., 2004).

One factor that has received little attention in the study of the quaternary ammonium block in $\mathrm{K}$ channels is the involvement of electrostatic charges on the channel surface, which can certainly affect the interaction of charged blockers like quaternary amines. Indeed, TEA block of frog node $\mathrm{K}$ channels is increased in low-ionic strength solutions or in solutions with reduced $\mathrm{Ca}^{2+}$ concentration (Mozhayeva \& Naumov, 1972). TEA block of a $\mathrm{Ca}^{2+}$-activated $\mathrm{K}$ channel is sensitive to solution ionic strength, and a chemically induced neutralization of negative surface charges reduces this ionic strength dependence (MacKinnon, Latorre \& Miller, 1989). The pHdependent TEA block of Kv1.1 channels is sensitive to changes in bulk solution ionic strength (Bretschneider et al., 1999). Finally, we have shown that TEA block of Shaker K channels is sensitive to bulk solution ionic strength and that block by a trivalent TEA analog (gallamine) is even more sensitive (Quinn \& Begenisich, 2001). The results of these various studies suggest a role for surface electrostatics in quaternary ammonium block of $\mathrm{K}$ channels.

Kv2.1 channels are rather unique among $\mathrm{K}$ channels in that they have a relatively low affinity for TEA in spite of possessing an aromatic amino acid at the TEA hot spot that confers high-affinity block to other $\mathrm{K}$ channels. They are unique also in having two lysine residues in the outer vestibule that are not conserved in other $\mathrm{K}$ channels. These lysine residues have a powerful effect on TEA block of these channels (Immke \& Korn, 2000; Immke et al., 1999). We have investigated the mechanism by which the positively charged side chains of these lysines affect TEA block. We tested the ionic strength dependence of block by TEA and the trivalent TEA analog gallamine as a way to probe for surface electrostatic effects. Kv3.1 channels have a high affinity for TEA and do not contain the Kv2.1 lysine residues noted above (Grissmer et al., 1994; Taglialatela et al., 1991). We found that, like Shaker K channels, quaternary amine block of Kv3.1 channels was ionic strengthdependent but block of wild-type Kv2.1 channels with the lysine residues was not. We also found that Kv2.1 channels were insensitive to gallamine. Neutralization of one or the other of the nonconserved lysine residues in Kv2.1 channels conferred ionic strength sensitivity to TEA block of Kv2.1 channels. Neutralization of both lysine side chains produced channels that were blocked by gallamine, and this block was more sensitive to solution ionic strength than block by TEA. Thus, surface electrostatics appears to play a role in TEA block of some, but not all, $\mathrm{K}$ channels depending on the location of some particular charged residues.

\section{Materials and Methods}

\section{K Channel Constructs}

Several $\mathrm{K}$ channel constructs were used in this study, including wild-type Kv2.1 and Kv3.1 channels (Chan Test Inc., Cleveland, $\mathrm{OH})$. Three mutant Kv2.1 channels were also investigated: K356F, $\mathrm{K} 382 \mathrm{Q}$ and the double mutant $\mathrm{K} 356 \mathrm{~F} / \mathrm{K} 382 \mathrm{Q}$. The replacement amino acids were introduced into the Kv2.1 clone using a two-step polymerase chain reaction (PCR) protocol and the resulting mutants analyzed by DNA sequencing.

\section{OOCyte Isolation And Microinjection}

Xenopus laevis oocytes were maintained as described by Goldin (1992). Isolated ovarian lobes were rinsed with $\mathrm{Ca}^{2+}$-free OR-2 solution (in $\mathrm{mm}$ ): $82.5 \mathrm{NaCl}, 2.5 \mathrm{KCl}, 1 \mathrm{MgCl}_{2}$ and 5 4-(2-hydroxyethyl)-1-piperazineethanesulfonic acid (HEPES; pH 7.6, $\mathrm{NaOH}$ ) and then defolliculated by incubation for 60-90 min with $2 \mathrm{mg} / \mathrm{ml}$ collagenase type 1A (Sigma, St. Louis, MO). Cleaned oocytes were transferred and maintained for $2 \mathrm{~h}$ in ND-96 solution (in mM): $96 \mathrm{NaCl}, 2 \mathrm{KCl}, 1.8 \mathrm{CaCl}_{2}, 1 \mathrm{MgCl}_{2}, 5$ HEPES and 2.5 Na-pyruvate ( $\mathrm{pH} 7.6, \mathrm{NaOH})$ before injection of mRNA coding for the channel construct of interest. Injected oocytes were transferred to multiwell tissue culture plates and incubated at $18^{\circ} \mathrm{C}$ in ND-96 solution supplemented with $100 \mathrm{U} / \mathrm{ml}$ penicillin and $100 \mu \mathrm{g} / \mathrm{ml}$ streptomycin. Animal (frog) handling and care utilized methods consistent with the National Institutes of Health's Guide for the Care and Use of Laboratory Animals.

\section{Electrophysiological ReCORDings}

$\mathrm{K}$ channel currents were recorded 1-5 days after mRNA injection. Recordings were made at room temperature $\left(20-22^{\circ} \mathrm{C}\right)$ using the 
cut-open oocyte voltage-clamp apparatus (model CA-1B; Dagan, Minneapolis, MN). Connections to the different compartments were made with glass capillaries containing $75 \mu \mathrm{m}$ platinum wires and filled with a $1 \mathrm{~m} \mathrm{NaCl}, 3 \%$ agar solution. The experimental chamber (ELV-1, Dagan) was modified to include a low-volume $(80 \mu \mathrm{l})$ chamber insert. The low chamber volume and high flow rates $(\sim 1 \mathrm{ml} / \mathrm{min})$ produced efficient solution exchange. The measured difference in junction potential for the two experimental solutions (see below) was $<2 \mathrm{mV}$, so no correction to membrane voltages was made.

Recording electrodes were made either of 1 BBL glass with filament $(1.5 \mathrm{~mm}$ outer diameter; World Precision Instruments, Sarasota, Fl) or of GC-150F glass (Warner Instrument, Hamden, CT). Electrodes had tip diameters of $\sim 2 \mu \mathrm{m}$ and were filled with a $3 \mathrm{~m} \mathrm{KCl}$ solution. Data acquisition was performed using a 12-bit analog/digital converter controlled by a personal computer. Current records were filtered at $5 \mathrm{kHz}$. Series resistance compensation was used: a mean value of $0.74 \pm 0.05$ (standard error of them mean, SEM) $\mathrm{k} \Omega$ in normal-ionic strength solutions and $2.2 \pm 0.11 \mathrm{k} \Omega$ in reduced-ionic strength solutions $(n=50)$.

Electrical access to the internal compartment was achieved by $0.125 \%$ saponin treatment in a solution of (in mM) $100 \mathrm{KCl}$ and 10 HEPES (pH 7.4, $\mathrm{N}$-methyl-D-glucamine [NMDG]). The external solution was (in mM) $140 \mathrm{NaCl}, 2 \mathrm{CaCl}_{2}, 10 \mathrm{KCl}, 10$ HEPES ( $\mathrm{pH}$ 7.4, NMDG). An external solution of reduced ionic strength was used that consisted of (in mM) $35 \mathrm{NaCl}, 0.5 \mathrm{CaCl}_{2}, 10 \mathrm{KCl}, 10$ HEPES ( $\mathrm{pH} 7.4$, NMDG). The osmolarity of this solution was matched to that of normal ionic strength by addition of $216 \mathrm{~mm}$ glucose. In order to test for possible effects of glucose beyond simply maintaining solution osmolarity, we also used mannitol and sucrose. We found that the ionic strength sensitivity of both TEA and gallamine block was the same regardless of which agent was used for osmotic balance. Osmolarity of all solutions was verified using a vapor pressure osmometer (Wescor, Logan, UT). Addition of TEA-Cl was made by substitution with $\mathrm{NaCl}$, which maintained solution ionic strength. Gallamine-I $\mathrm{I}_{3}$ (1,2,3-tris[2-triethylammonium ethoxy]benzene triiodide; Sigma) was added to the external solution with no compensation for changes in solution ionic strength.

Oocytes were clamped at a holding potential of $-80 \mathrm{mV}$, and 80 -ms test pulses were applied to elicit channel current. Channel block by TEA or gallamine was computed by calculating the steady-state current recorded at the end of each pulse as a fraction of the average current recorded before application and following washout of the blocker. Only those results with at least $90 \%$ recovery from block were considered. Concentration-response relationships were constructed by plotting the mean fraction of current blocked at a test potential of $+40 \mathrm{~m} V$. These were fit by the standard binding isotherm:

Fraction Blocked $=B_{\max } \frac{[B]}{[B]+K_{a p p}}$

where $[B]$ is the concentration of TEA or gallamine, $B_{\max }$ is the maximal fraction of current blocked and $K_{a p p}$ is the concentration that blocks half the maximal current.

We used the crystal structure of the KcsA K channel (RCSB Protein Data Bank entry 1J95) as a template for the Kv2.1 channel (Doyle et al., 1998). The KcsA residues in the outer vestibule were mutated to their Kv2.1 counterparts with the DeepView/SwissPdbViewer, version 3.7 (http://ca.expasy.org/spdbv/). The molecular views of the $\mathrm{K}$ channel structures were generated with ViewerLite 4.2 for Windows (Accelrys, San Diego, CA; http:// www.accelrys.com/). The electrostatic surface view was also generated with ViewerLite using Gasteiger charges.

\section{Results}

\section{Charged Residues in the Outer Vestibule of K Channels}

Figure 1 illustrates two views of the outer vestibule of $\mathrm{K}$ channels. Shown are schematics based on the crystal structure of the bacterial $\mathrm{K}$ channel KcsA, but all crystallized $\mathrm{K}$ channels, including the mammalian voltage-gated $\mathrm{K}$ channel Kv1.2, share a common structure for this outer part of the channel (Long et al., 2005). On the left is a view from above the channel, and the right part of the figure illustrates a side view with two of the subunits removed. Shown below the structures is an alignment of the amino acids in the outer vestibule and selectivity filter of Shaker, Kv2.1 and Kv3.1 channels. As noted in the Introduction, an aromatic residue at Shaker position 449 confers high-affinity TEA block. Replacement of the native threonine at position 449 in Shaker channels to a tyrosine renders the channel highly sensitive to TEA (Kavanaugh et al., 1991; MacKinnon \& Yellen, 1990). Both Kv2.1 and Kv3.1 channels have a tyrosine at this position (shown in green).

All three channels contain several negative amino acids in this vestibule region (indicated in red in the alignment). Kv2.1 channels are unique in having two positively charged amino acids (K382 and K356) in the outer vestibule (blue in the alignment and illustrated as space-filling atoms in the structural diagrams). The influence of these lysine residues on TEA block is an important part of the present study.

\section{Ionic Strength-dependent Block of Kv3.1 ${\text { Channels by } \text { TEA }^{+} \text {and Gallamine }}^{3+}$}

As described above, TEA block of Shaker K channels is sensitive to solution ionic strength, and block by a trivalent TEA analog, gallamine, is even more sensitive (Quinn \& Begenisich, 2001). These results demonstrate that negative charges on the surface of the channel contribute to an electrostatic potential that influences block by these charged molecules. As a first step toward identifying the amino acids that could contribute to this type of surface electrostatics, we tested the ionic strength dependence of TEA and gallamine block of two other K channels: Kv3.1 and Kv2.1.

Shown in the upper part of Figure 2A are raw current traces from an oocyte expressing Kv3.1 channels. TEA $(50 \mu \mathrm{M})$ produced a small (near $25 \%$ ) block of Kv3.1 channels in a normal-ionic strength solution (see Materials and Methods) but almost a $50 \%$ block when applied in a solution of low ionic strength. The lower panel illustrates the concentration dependence of current block, measured at +40 $\mathrm{m} V$, at normal and low ionic strength. The lines are fits of equation 1 to the data with a $K_{a p p}$ value of 0.14 

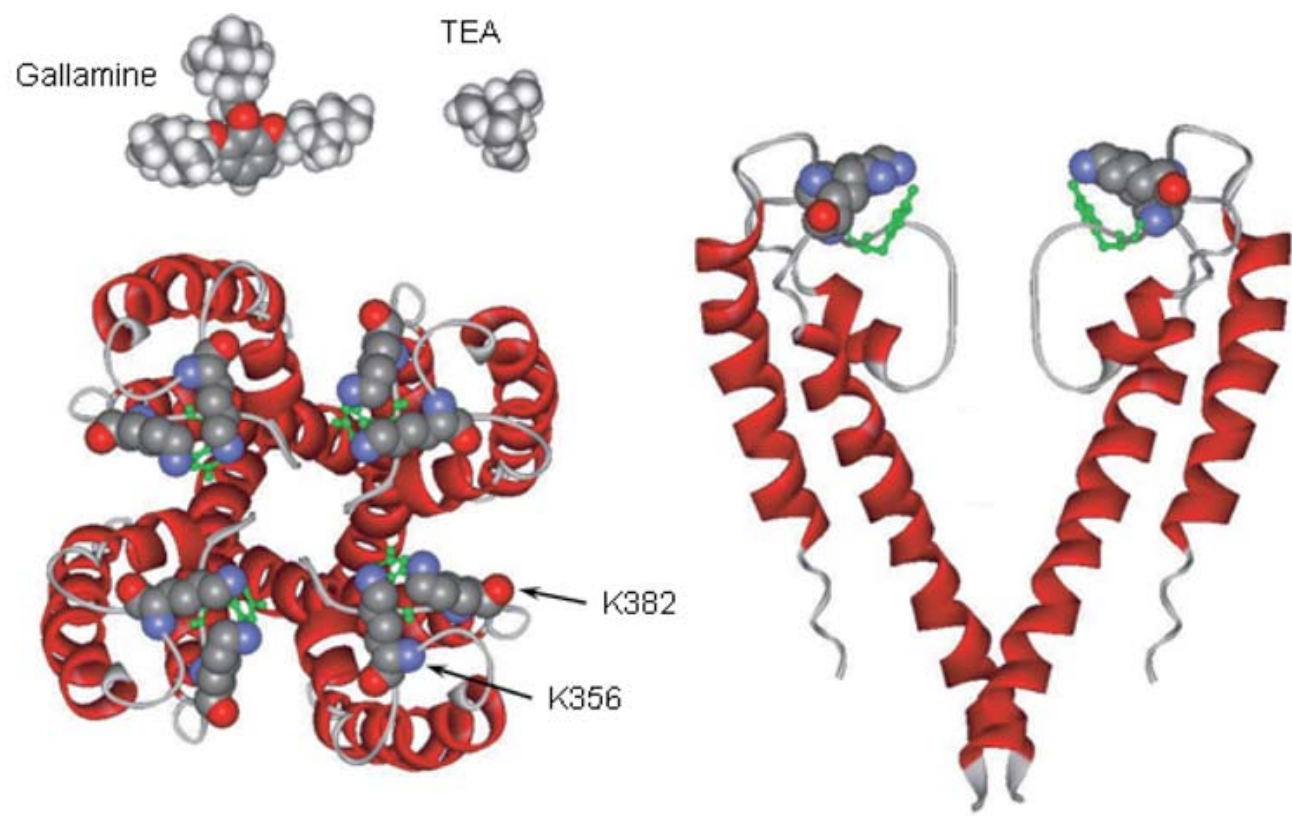

Shaker EAGSENSE

FKSI PDAFWNAVNTMTTVGYGDMTP VGVWGK 456

KV2.1 EKDEDDTK

EKSI PASEINATITMTTVGYGDIYP KTLLGK 387

KV3.1 ERIGAQPNDPSASEHTHEKNI PIGEWAAVVTMTTLGYGDMYP QTMSGM 404

Fig. 1. Sequence and structure of the outer vestibule of K channels. Bottom, Amino acid sequence alignment of three $\mathrm{K}$ channels in the region that forms much of the channel pore outer vestibule. In blue are the two lysine residues (K356 and K382) in Kv2.1 channels that are a major focus of this study. Kv2.1 and Kv3.1 channels have a tyrosine amino acid (shown in green) at the position that is important for highaffinity TEA block (see text). In red are several amino acids (aspartate and glutamate) in the vestibule area that have negatively charged side chains. Top left, Structures of gallamine and TEA. Bottom left, Top-view structure of KcsA. The fourfold symmetry of the channel (in ribbon form) is apparent in this view. The Kv2.1 K356 and K382 lysine residues are shown with space-filling atoms (without hydrogens). The tyrosine important for TEA binding is shown with "ball and stick" atoms in green. Right, Side view of the channel with two subunits removed for clarity. Apparent are the outer vestibule and narrow selectivity filter. As in the top view, the relevant lysines and tyrosines are shown with space-filling and ball-and-stick atoms, respectively.

mM for TEA block in the normal-ionic strength solution, well within the $0.09-0.2 \mathrm{~mm}$ range previously reported for TEA block of this $\mathrm{K}$ channel (Grissmer et al., 1994; Jarolimek et al., 1995; Taglialatela et al., 1991). TEA was more effective in the low-ionic strength solution with a $K_{a p p}$ value of 0.055 $\mathrm{mM}$, a 2.5 -fold increase in apparent TEA affinity in the low-ionic strength solution.

The upper part of Figure 2B contains raw currents illustrating block by $20 \mu \mathrm{M}$ gallamine at normal and low ionic strength. This concentration of gallamine blocked very little current at normal ionic strength $(<20 \%)$, but more than half the current was blocked when the gallamine was applied in the lowionic strength solution. The lower part of Figure 2B shows the concentration dependence of block by gallamine, measured at $+40 \mathrm{mV}$, at normal and low ionic strength. The lines are fits of equation 1 to the data with $K_{a p p}$ values of $0.066 \mathrm{~mm}$ at normal and $0.017 \mathrm{~mm}$ at low ionic strength, a 3.9-fold increase in apparent gallamine affinity in the low-ionic strength solution. Similar results have been observed for gallamine block of Shaker K channels (Quinn \& Begenisich, 2001).

Thus, quaternary amine block of Kv3.1 channels, like Shaker K channels, was sensitive to solution ionic strength and this sensitivity was greater for trivalent than monovalent blocking ions. These results demonstrate that Kv3.1, like Shaker channels, contain charged residues that contribute to a negative electrostatic potential that influences block by these charged molecules. Our further investigations indicated that not all channels share this property, as demonstrated in Figure 3.

\section{$\mathrm{TEA}^{+}$Block of Kv2.1 Channels Was Not} Sensitive to Ionic Strength

The upper part of Figure 3 illustrates TEA block of currents recorded from Kv2.1 channels. The raw current records show that, in this oocyte, block by $5 \mathrm{~mm}$ TEA was about 35\%, independent of the solution ionic strength. The lower part of the figure demonstrates the dose-response relation for block of 


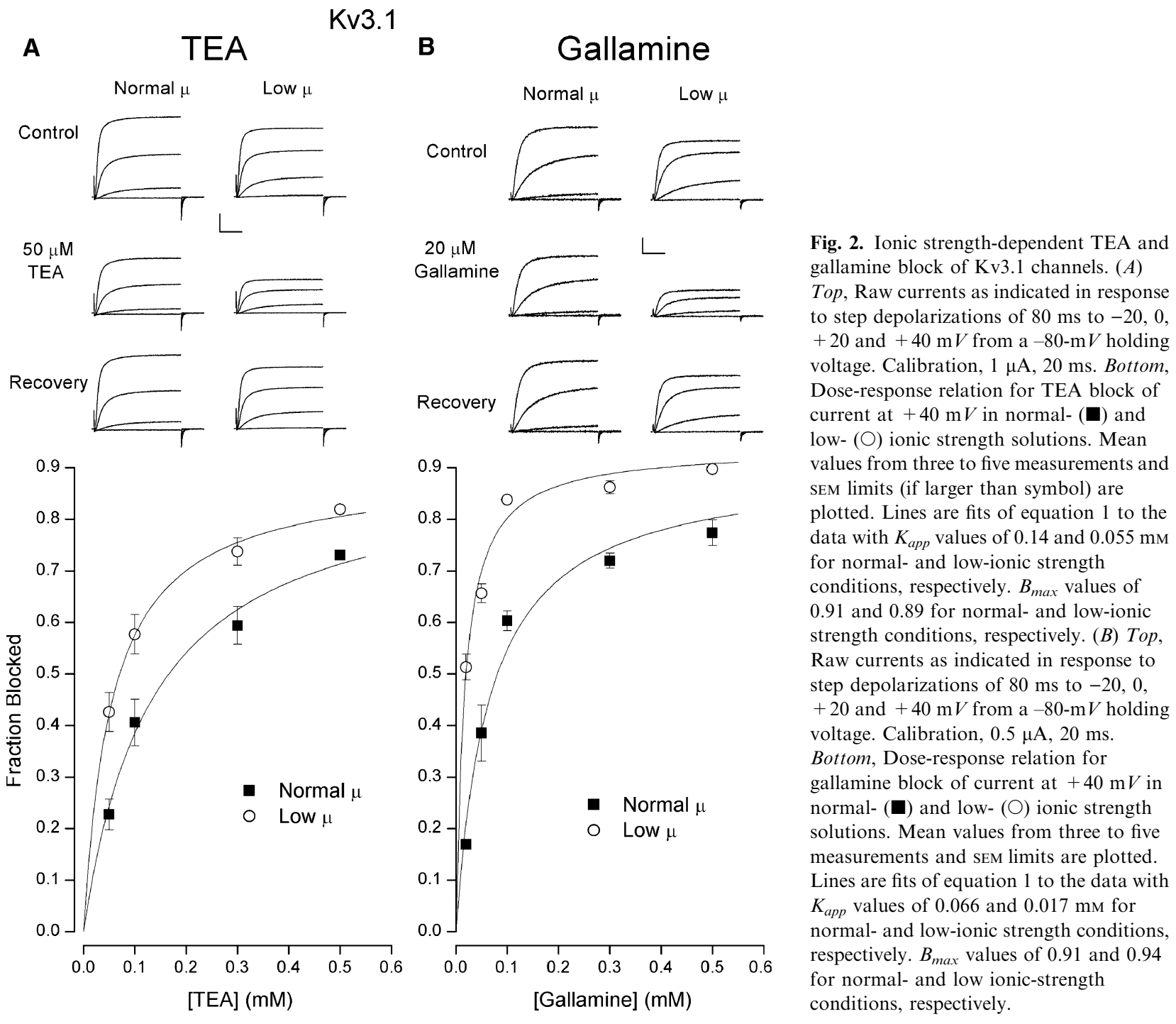

the ionic current at $+40 \mathrm{~m} V$ from many oocytes in the normal- and low-ionic strength solutions. Clearly, TEA block of Kv2.1 channels was not ionic strengthdependent and well described by a $K_{a p p}$ value of 5.1 $\mathrm{mm}$. This $K_{a p p}$ value is quite similar to the $4.2-4.5 \mathrm{~mm}$ values previously reported for these channels (Immke et al., 1999; Taglialatela et al., 1991) and illustrates the fact that these Kv2.1 channels are almost 40 -fold less sensitive to TEA block than other $\mathrm{K}$ channels in spite of the fact that they contain the aromatic amino acids normally associated with high-affinity TEA block. In addition, unlike Shaker and Kv3.1 channels, TEA block of Kv2.1 channels was not sensitive to solution ionic strength.

Kv2.1 channels also differed from Shaker (T449Y) and Kv3.1 channels with respect to block by gallamine. We could detect no significant block of Kv2.1 channels by this trivalent TEA analog. In the normal-ionic strength solution, there was an average $3.5 \%$ increase in current in the presence of $5 \mathrm{~mm}$ gallamine, but this was not significantly different from zero $(n=3)$. Similar results showing no significant block were obtained with $10 \mathrm{~mm}$ gallamine and with the low-ionic strength solution.

Our low-ionic strength solution contained onefourth the normal $\mathrm{Na}^{+}$and $\mathrm{Ca}^{2+}$ concentrations than the normal-ionic strength solution. In addition to changes in ionic strength induced by alterations in the monovalent ion concentrations, changes in $\mathrm{Ca}^{2+}$ concentrations are known to shift the voltage dependence of $\mathrm{K}$ channel gating but do not block these channels (e.g., Begenisich, 1975; Frankenhaeuser \& Hodgkin, 1957; Mozhayeva \& Naumov, 1972). It is because of this effect of alterations in $\mathrm{Na}^{+}$ and $\mathrm{Ca}^{2+}$ concentrations that we measured TEA and gallamine block at $+40 \mathrm{mV}$, a voltage at which there are minimal gating changes. Nevertheless, it is worthwhile to demonstrate that these gating shifts do not affect our measurement of TEA block in the normal- and low-ionic strength solutions. Thus, in a separate set of experiments, we measured TEA block of Kv3.1 and Kv2.1 channels at several voltages in 


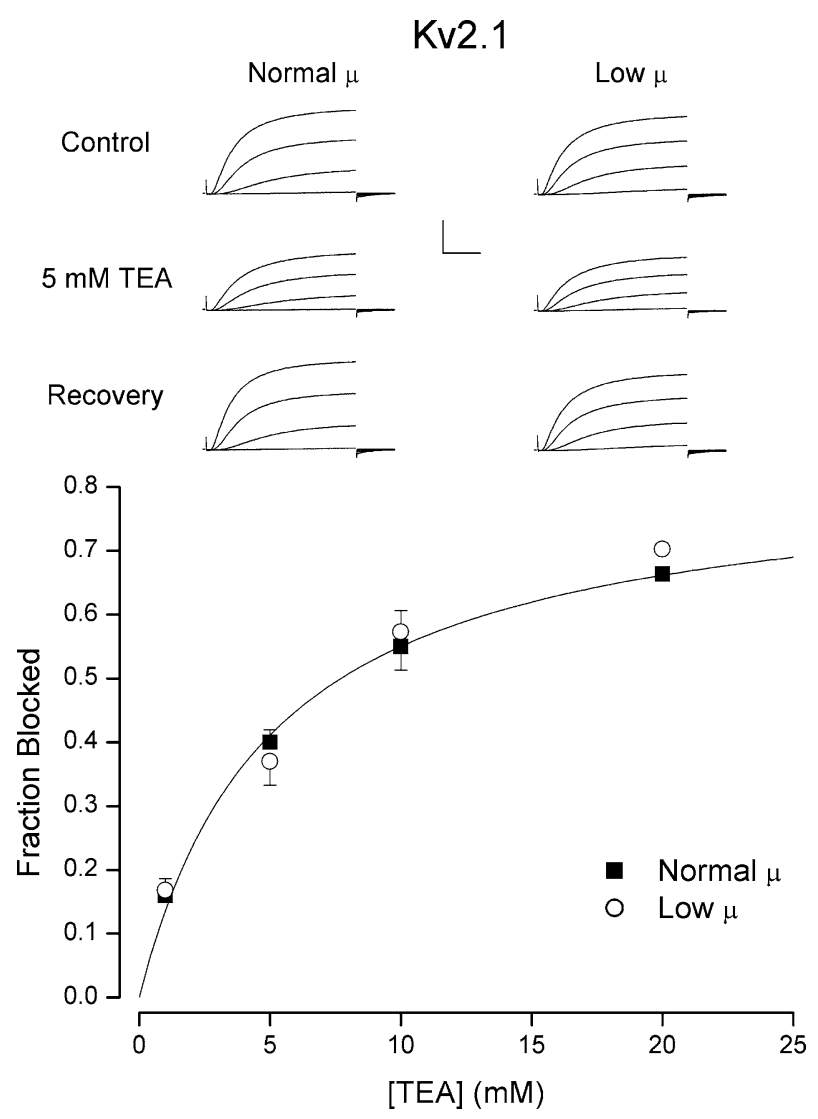

Fig. 3. Ionic strength-dependent TEA block of Kv2.1 channels. $T o p$, Raw currents as indicated in response to step depolarizations of $80 \mathrm{~ms}$ to $-20,0,+20$ and $+40 \mathrm{mV}$ from a $-80-\mathrm{m} V$ holding voltage. Calibration, $2 \mu \mathrm{A}, 20 \mathrm{~ms}$. Bottom, Dose-response relation for TEA block of current at $+40 \mathrm{~m} V$ in normal- $(\mathbf{\square})$ and low- $(\mathrm{O})$ ionic strength solutions. Mean values from 4-10 measurements and SEM limits (if larger than symbols) are plotted. Line is a fit of equation 1 to the pooled normal- and low-ionic strength data with $K_{a p p}$ and $B_{\max }$ values of $5.1 \mathrm{~mm}$ and 0.83 , respectively.

the two ionic strength solutions. As can be seen in Figure 4, there is a slight voltage dependence to the TEA block of Kv3.1 channels and little or no voltage dependence to block of Kv2.1 channels, consistent with previous observations on these channels (Hartmann et al., 1991; Taglialatela et al., 1991). Importantly, these results show that the ionic strength dependence of TEA block was independent of test potential, so our use of $+40 \mathrm{~m} V$ introduced no bias into the analysis.

As noted in Introduction, the low Kv2.1 sensitivity to TEA is surprising since, like Kv3.1, this channel contains the tyrosine residue at the "hot spot" necessary for high-affinity TEA binding (see Fig. 1). This issue has been discussed previously (Taglialatela et al., 1991), and one possibility that those authors suggested is that the positively charged side chain of the nearby K382 (see Fig. 1) could "repulse TEA" and so reduce Kv2.1 TEA sensitivity. If there were a significant through-space electrostatic repulsion of TEA by the charged side chain of K382, then that repulsion should increase in a low-ionic strength solution. The data presented in Figure 3, however, show that this did not occur.

We suggest that there is little or no net surface charge on wild-type Kv2.1 channels in a position to influence TEA block, so ionic strength should have no effect, as the data demonstrated. If this is correct, then removing some of the positive charge should shift the balance to a net negative surface charge and confer ionic strength dependence to TEA block. Thus, we mutated K382 in Kv2.1 to the neutral glutamine that occupies the analogous location in Kv3.1 channels. The action of TEA on these mutant channels is illustrated in Figure 5A.

\section{Positive Charge Neutralization in Kv2.1 Channels}

As illustrated in Figure 5A, TEA blocked K382Q Kv2.1 channels with an apparent $K_{a p p}$ value near 5 $\mathrm{mm}$ in the normal-ionic strength solution. TEA was more effective when applied in the low-ionic strength solution: block was described by a $K_{a p p}$ value near $2 \mathrm{~mm}$. Thus, as predicted, removal of positive charge altered the electrostatic environment in the vestibule such that TEA block became sensitive to solution ionic strength. These K382Q channels remained resistant to block by $5 \mathrm{~mm}$ gallamine (not significantly different from zero, $n=3$ ).

Inspection of the likely topology of the outer pore of Kv2.1 channels (Fig. 1) suggests that the other positively charged amino acid unique to Kv2.1 (K356) may also be in a position to electrostatically effect TEA block. Thus, we tested TEA block of Kv2.1 channels with the lysine at position $356 \mathrm{mu}-$ tated to the equivalent residue in Shaker (phenylalanine). Figure 5B shows TEA block of these K356F channels. As can be seen, replacement of the charged K356 residue, like neutralization of K382, resulted in a channel with ionic strength-dependent TEA block: $K_{\text {app }}$ values of 6.1 and $2.8 \mathrm{~mm}$ in the normal- and lowionic strength solutions, respectively. This construct was also quite insensitive to block by gallamine: $5 \mathrm{~mm}$ inhibited only $4.4 \pm 0.5 \%$ of the current at $+40 \mathrm{mV}$.

We also tested the ionic strength dependence of block of channels with both charged lysine residues neutralized. Figure 6A shows that TEA block of the double mutant $\mathrm{K} 356 \mathrm{~F} / \mathrm{K} 382 \mathrm{Q}$ channels was sensitive to solution ionic strength. Figure $6 \mathrm{~B}$ demonstrates that these double mutant channels, unlike wild-type channels or channels with only a single neutralization, could be blocked by millimolar concentrations of gallamine. In normal-ionic strength solution, the $K_{a p p}$ for gallamine block was about $1.2 \mathrm{~mm}$; in reduced-ionic strength solution, it was about $0.13 \mathrm{~mm}$. This almost 10-fold increase in gallamine block with ionic strength change was even larger than that seen in Kv3.1 channels (Fig. 2B). 


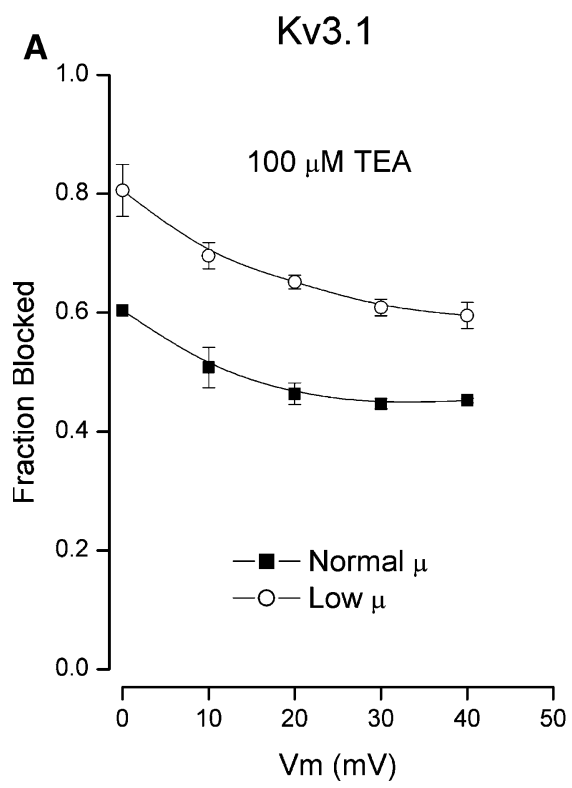

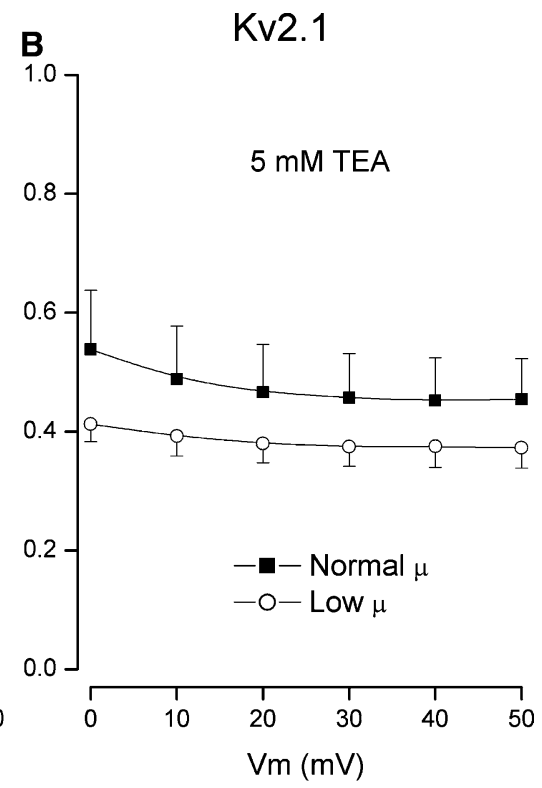

Fig. 4. Voltage dependence of TEA block of Kv3.1 and Kv2.1 channels. (A) Fraction of Kv3.1 channel current blocked by $100 \mu \mathrm{M}$ TEA at the indicated membrane potentials in normal- $(\square)$ and low- $(O)$ ionic strength solutions. $(B)$ Fraction of Kv2.1 channel current blocked by $5 \mathrm{~mm}$ TEA at the indicated membrane potentials in normal- $(\boldsymbol{\square})$ and low- $(\bigcirc)$ ionic strength solutions.
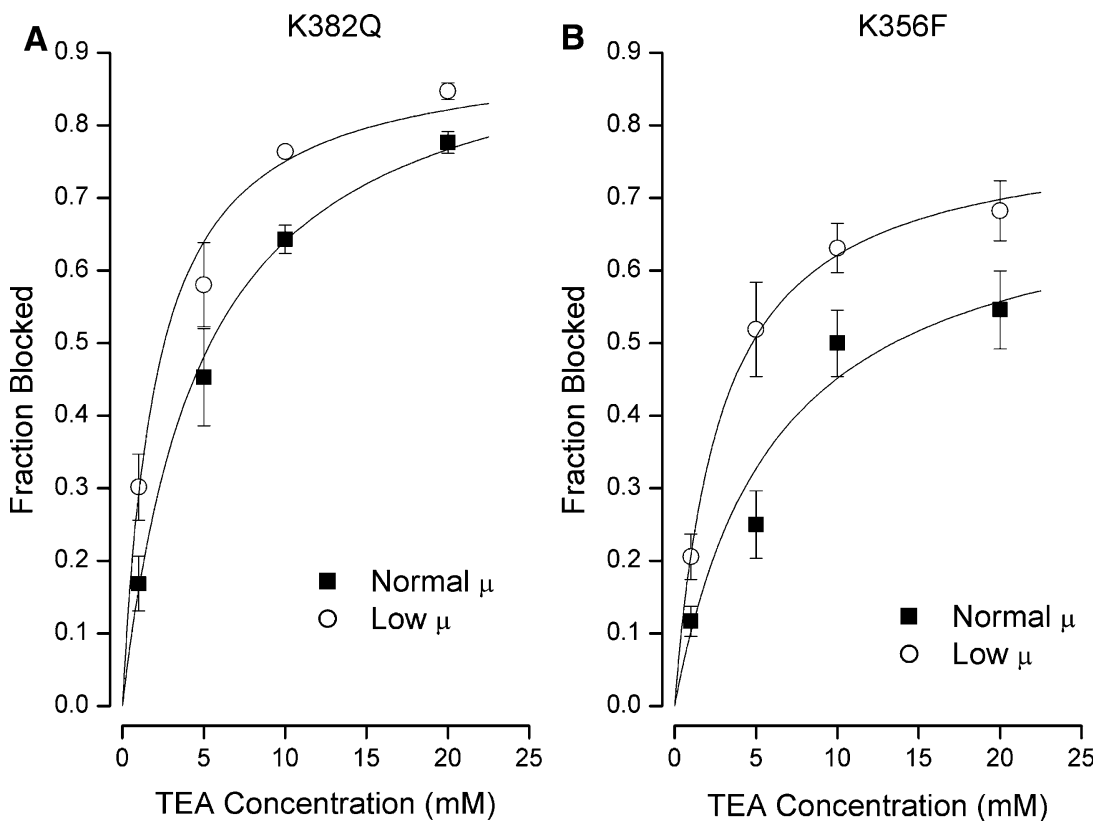

Fig. 5. Ionic strength-dependent TEA block of mutant Kv2.1 channels. (A) Dose-response relation for TEA block of current (at $+40 \mathrm{~m} V$ ) from Kv2.1 K382Q channels in normal- $(\mathbf{\square})$ and low- $(O)$ ionic strength solutions. Mean values from three measurements and sEM limits (if larger than symbols) are plotted. Line is a fit of equation 1 to the data with $K_{a p p}$ values of 4.9 and $2.1 \mathrm{~mm}$ for normal- and low-ionic strength conditions, respectively. $B_{\max }$ values of 0.96 and 0.91 for normal- and low-ionic strength conditions, respectively. $(B)$ Dose-response relation for TEA block of current (at $+40 \mathrm{~m} V$ ) from Kv2.1 K356F channels in normal- $(\mathbf{\square})$ and low- $(\bigcirc)$ ionic strength solutions. Mean values from four to six measurements and SEM limits are plotted. Line is a fit of equation 1 to the data with $K_{a p p}$ values of 6.1 and $2.8 \mathrm{~mm}$ for normal- and low- ionic strength conditions, respectively. $B_{\max }$ values of 0.73 and 0.80 for normal- and low-ionic strength conditions, respectively.

\section{Discussion}

TEA block of some $\mathrm{K}$ channels is sensitive to ionic strength changes (MacKinnon et al., 1989; Quinn \& Begenisich, 2001), indicating that surface electrostatics plays a role in the permeation and pharmacological properties of such channels. We showed here that this property is shared by Kv3.1 channels. In contrast, TEA block of wild-type Kv2.1 channels was insensitive to solution ionic strength. Kv2.1 channels are also unusual in having a relatively low TEA affinity in spite of having the type of amino acid at the critical position that normally confers high TEA affinity (Kavanaugh et al., 1991; MacKinnon \& 


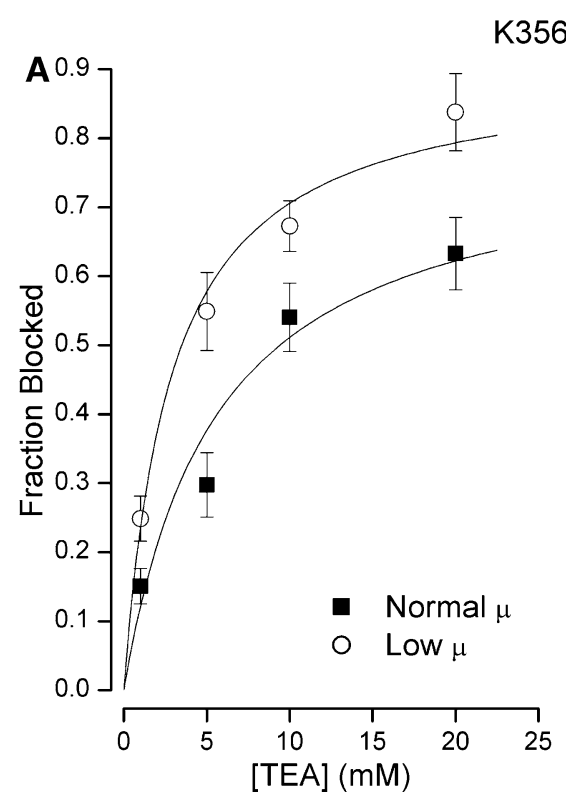

K356F/K382Q

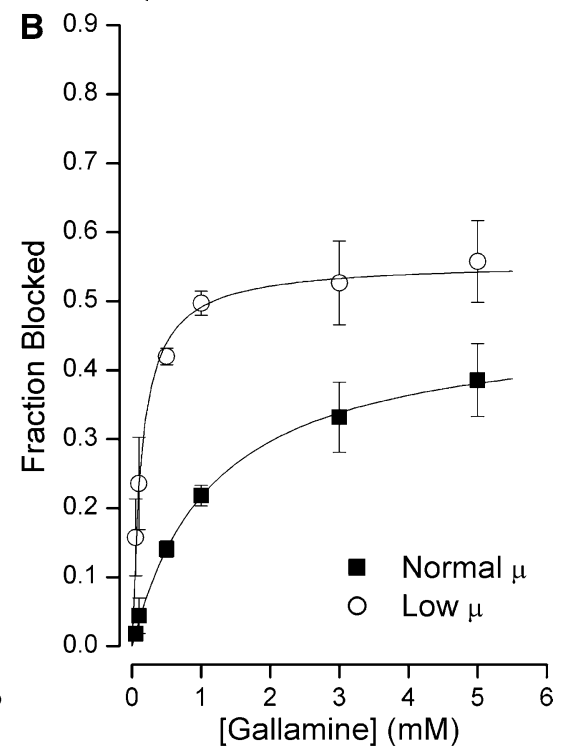

Fig. 6. Ionic strength-dependent TEA and gallamine block of K356F/K382Q Kv2.1 channels. ( $A$ ) Dose-response relation for TEA block of current (at $+40 \mathrm{mV}$ ) in normal- $(\mathbf{\square})$ and low- $(O)$ ionic strength solutions. Mean values from three to six measurements and sEM limits are plotted. Line is a fit of equation 1 to the data with $K_{a p p}$ values of 5.6 and $2.8 \mathrm{~mm}$ for normal- and low-ionic strength conditions, respectively. $B_{\max }$ values of 0.80 and 0.91 for normal- and low-ionic strength conditions, respectively. $(B)$ Dose-response relation for gallamine block of current (at $+40 \mathrm{mV}$ ) in normal- $(\mathbf{\square})$ and low- $(O)$ ionic strength solutions. Mean values from three to six measurements and sEm limits (if larger than symbols) are plotted. Line is a fit of equation 1 to the data with $K_{a p p}$ values of 1.2 and $0.13 \mathrm{~mm}$ for normal- and low-ionic strength conditions, respectively. $B_{\max }$ values of 0.47 and 0.56 for normal- and low-ionic strength conditions, respectively.

Yellen, 1990). We showed that Kv2.1 channels were also unusual in being insensitive to the trivalent TEA analog gallamine.

Inspection of the crystal structures of the outer vestibule of $\mathrm{K}$ channels and the specific amino acid composition of Shaker, Kv2.1 and Kv3.1 channels (see Fig. 1) reveals that the noted pharmacological differences of Kv2.1 channels could result from the presence of charged lysine residues not represented in Kv3.1 or Shaker channels. Neutralization of either K356 or K382 in Kv2.1 conferred an ionic strength-sensitive TEA block to these channels (Fig. 5), but these mutant channels remained very insensitive to block by gallamine. Kv2.1 channels with both positively charged lysines neutralized attained a reasonable sensitivity to gallamine block, which was particularly sensitive to solution ionic strength (Fig. 6).

The lack of ionic strength sensitivity of TEA block of Kv2.1 channels indicates that these channels, in contrast to Shaker and Kv3.1 channels, have little or no net charge on the outer vestibule in a position to affect TEA block. If so, then neutralization of the charged lysines not represented in Shaker and Kv3.1 channels should result in a net negative surface charge and thus confer ionic strength sensitivity to TEA block on the mutant Kv2.1 channels. This was the result that was obtained (Fig. 4). Figure 7 illustrates the outer entrance of the KcsA channel pore which has been "mutated" to contain Kv2.1 amino acids. Shown is the electrostatic potential arising from the charged amino acids as it is mapped onto the protein. Negative potentials are in red and positive potentials are in blue. The most prominent charged side chains are identified, and it is clear that K356 and K382 sit quite close to the pore entrance but that several negatively charged amino acids are also nearby. The lack of ionic strength dependence of TEA block of Kv2.1 indicates a negligible surface potential in a position to affect TEA block. Thus, it is likely that that the two lysine residues near the pore (K382 and K356) and the one farther away (K350) are sufficient to balance the electric field from the four negatively charged amino acids in the vicinity (D351, E352, D353, D354). According to this picture, mutating either or both of K356/K382 would cause the near zero electrostatic potential of the wild-type channel to shift negative, accounting for the ionic strength dependence of the TEA block of these mutant channels.

The "uncovering" of negative charges caused by replacing the lysines with uncharged amino acids would be expected not only to confer ionic strength dependence on TEA block (as observed) but also to increase the apparent affinity for TEA block. While these charge-mutant channels did exhibit an ionic strength-dependent TEA block, their TEA sensitivities were essentially the same as the wild-type channel. It is possible that the mutations also produced some minor structural alteration in the outer 


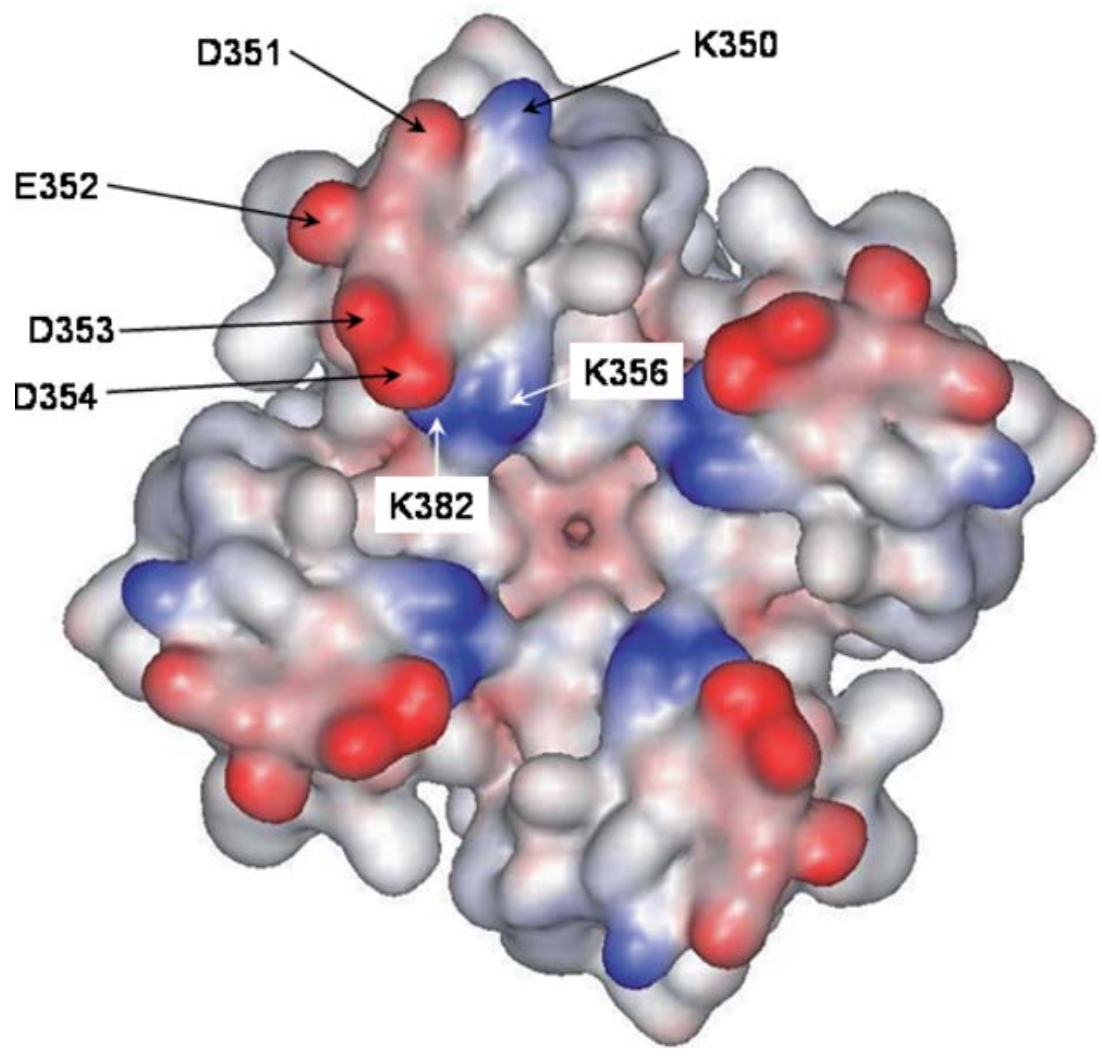

Fig. 7. Electrostatic potential map of the outer vestibule of Kv2.1 channels. Top view of the channel with the pore in the middle; the fourfold symmetry of these $\mathrm{K}$ channels is apparent. Electrostatic potentials computed as described in Materials and Methods and mapped onto the surface of the Kv2.1 channel.

Negatively charged amino acid side chains are red, and blue represents positive charges. The two lysine residues that are the focus of this work (K336 and K382) as well as the other exposed charged amino acids are indicated in one of the four channel subunits.

vestibule that decreased the TEA affinity, masking the apparent increase expected with a more negatively charged vestibule. This view is supported by the fact that replacement of these lysines with neutral amino acids different from those used here (K382V, K356G) does, in fact, increase TEA affinity (Immke et al., 1999).

Our data also suggest that at least one component of the gallamine sensitivity of $\mathrm{K}$ channels may be the net charge in the outer vestibule. The intrinsic gallamine affinity for $\mathrm{K}$ channels may be relatively low, but the negatively charged vestibules intrinsic to, for example, Shaker and Kv3.1 channels would increase the local gallamine concentration and thus overcome the intrinsic low affinity. The essentially neutral vestibule of wild-type Kv2.1 channels cannot be occupied by gallamine at millimolar levels. Even the mild negativity of K356F and K382Q Kv2.1 channels is not sufficient to allow reasonable block by gallamine. However, removal of both positively charged lysines (a total of eight positive charges on these tetrameric channels) apparently produces a sufficiently negative vestibule to provide for gallamine block. Consistent with this strongly negatively charged vestibule is the very large ionic strength dependence of gallamine block of these double mutant channels (Fig. 5B).

Another feature of the gallamine block of the double mutant Kv2.1 channels was the apparent saturation of block at levels well below 100\%
(Fig. 6B). We do not know the reason for this effect, but it is worth noting that, under some circumstances, TEA block of Kv2.1 channels displays this phenomenon (Hartmann et al., 1991; Immke \& Korn, 2000; Immke et al., 1999). Such a result would be observed if the channels had both high and very low affinity for these blocking ions. The outer vestibule of KcsA channels exists in two conformations controlled by $\mathrm{K}^{+}$levels (Zhou et al., 2001), and Shaker K channels exhibit both high- and low-affinity TEA block configurations also controlled by the $\mathrm{K}^{+}$levels (Thompson \& Begenisich, 2005). Future experiments will be required to test if Kv2.1 channels (or mutant Kv2.1 channels) exhibit such behavior with respect to gallamine block.

Finally, it should be noted that electrostatic potentials contribute to the block of other types of ion channels. For example, snail toxin peptide block of voltage-gated $\mathrm{Na}$ channels, like scorpion toxin block of K channels (MacKinnon et al., 1989), exhibits strong charge-dependent effects (Hui et al., 2002). While the details of block are different in these two examples, these and the results of the present study demonstrate the widespread importance of electrostatic potentials in the outer vestibule of ion channels.

In summary, the results reported here indicate that surface electrostatics may play a significant role in the pharmacology of $\mathrm{K}$ channels. Shaker and Kv3.1 channels have negative charges in the outer 
vestibule that contribute to TEA and gallamine block. Wild-type Kv2.1 channels have a lower TEA affinity than Kv3.1 and other channels even though these channels possess the aromatic amino acid necessary for high-affinity TEA block. Our results suggest that at least one reason for this is that Kv2.1 channels do not have the exposed negative charges that contribute to TEA block in Kv3.1 and Shaker channels, likely because these negative charges are rendered ineffective by the nearby positively charged side chains of lysines at positions 356 and 382. Gallamine cannot block Kv2.1 channels unless both lysines are neutralized, likely because this TEA analog has an intrinsically low affinity but is particularly sensitive to electrostatic potentials owing to its trivalent nature. A full understanding of the pharmacology of the outer vestibule of $\mathrm{K}$ channels will have to take into account the electrostatic charges in this structural region.

We thank Dr. Glenn Kirsch for providing the Kv2.1 and Kv3.1 clones. We are grateful to Jill Thompson for molecular biological and other technical assistance, for helpful discussions and for critically reading this manuscript.

\section{References}

Andalib, P., Consiglio, J.F., Trapani, J.G., Korn, S.J. 2004. The external TEA binding site and C-type inactivation in voltagegated potassium channels. Biophys. J. 87:3148-3161

Begenisich, T. 1975. Magnitude and location of surface charges on Myxicola giant axons. J. Gen. Physiol. 66:47-65

Bretschneider, F., Wrisch, A., Lehmann-Horn, F., Grissmer, S. 1999. External tetraethylammonium as a molecular caliper for sensing the shape of the outer vestibule of potassium channels. Biophys. J. 76:2351-2360

Crouzy, S., Berneche, S., Roux, B. 2001. Extracellular blockade of $\mathrm{K}^{+}$channels by TEA: Results from molecular dynamics simulations of the KcsA channel. J. Gen. Physiol. 118:207-218

Doyle, D.A., Morais Cabral, J., Pfuetzner, R.A., Kuo, A., Gulbis, J.M., Cohen, S.L., Chait, B.T., MacKinnon, R. 1998. The structure of the potassium channel: Molecular basis of $\mathrm{K}^{+}$ conduction and selectivity. Science 280:69-77

Frankenhaeuser, B., Hodgkin, A.L. 1957. The action of calcium on the electrical properties of squid axon. J. Physiol. 137:218-244

Goldin, A.L. 1992. Maintenance of Xenopus laevis and oocyte injection. Methods Enzymol. 207:266-279

Grissmer, S., Nguyen, A.N., Aiyar, J., Hanson, D.C., Mather, R.J., Gutman, G.A., Karmilowicz, M.J., Auperin, D.D., Chandy, K.G. 1994. Pharmacological characterization of five cloned voltage-gated $\mathrm{K}^{+}$channels, types $\mathrm{Kv1} 1,1.2,1.3,1.5$, and 3.1, stably expressed in mammalian cell lines. Mol. Pharmacol. 45:1227-1234

Hartmann, H.A., Kirsch, G.E., Drewe, J.A., Taglialatela, M., Joho, R.H., Brown, A.M. 1991. Exchange of conduction pathways between two related $\mathrm{K}^{+}$channels. Science 251:942944

Heginbotham, L., MacKinnon, R. 1992. The aromatic binding site for tetraethylammonium ion on potassium channels. Neuron 8:483-491

Hille, B. 2001. Ion Channels of Excitable Membranes. Sinauer Associates, Sunderland, MA
Hui, K., Lipkind, G., Fozzard, H.A., French, R.J. 2002. Electrostatic and steric contributions to block of the skeletal muscle sodium channel by mu-conotoxin. J. Gen. Physiol. 119:45-54

Immke, D., Korn, S.J. 2000. Ion-ion interactions at the selectivity filter. Evidence from $\mathrm{K}^{+}$-dependent modulation of tetraethylammonium efficacy in Kv2.1 potassium channels. J. Gen. Physiol. 115:509-518

Immke, D., Wood, M., Kiss, L., Korn, S.J. 1999. Potassiumdependent changes in the conformation of the Kv2.1 potassium channel pore. J. Gen. Physiol. 113:819-836

Jarolimek, W., Soman, K.V., Alam, M., Brown, A.M. 1996. Structure-activity relationship of quaternary ammonium ions at the external tetraethylammonium binding site of cloned potassium channels. Mol. Pharmacol. 49:165-171

Jarolimek, W., Soman, K.V., Brown, A.M., Alam, M. 1995. The selectivity of different external binding sites for quaternary ammonium ions in cloned potassium channels. Pfluegers Arch. 430:672-681

Jiang, Y., Lee, A., Chen, J., Cadene, M., Chait, B.T., MacKinnon, R. 2002. Crystal structure and mechanism of a calcium-gated potassium channel. Nature 417:515-522

Jiang, Y., Lee, A., Chen, J., Ruta, V., Cadene, M., Chait, B.T., MacKinnon, R. 2003. X-ray structure of a voltage-dependent $\mathrm{K}^{+}$channel. Nature 423:33-41

Kavanaugh, M.P., Hurst, R.S., Yakel, J., Varnum, M.D., Adelman, J.P., North, R.A. 1992. Multiple subunits of a voltagedependent potassium channel contribute to the binding site for tetraethylammonium. Neuron 8:493-497

Kavanaugh, M.P., Varnum, M.D., Osborne, P.B., Christie, M.J., Busch, A.E., Adelman, J.P., North, R.A. 1991. Interaction between tetraethylammonium and amino acid residues in the pore of cloned voltage-dependent potassium channels. J. Biol. Chem. 266:7583-7587

Long, S.B., Campbell, E.B., MacKinnon, R. 2005. Crystal structure of a mammalian voltage-dependent Shaker family $\mathrm{K}^{+}$ channel. Science 309:897-903

MacKinnon, R., Latorre, R., Miller, C. 1989. Role of surface electrostatics in the operation of a high-conductance $\mathrm{Ca}^{2+}$. activated $\mathrm{K}^{+}$channel. Biochemistry 28:8092-8099

MacKinnon, R., Yellen, G. 1990. Mutations affecting TEA blockade and ion permeation in voltage-activated $\mathrm{K}^{+}$channels. Science 250:276-279

Mozhayeva, G.N., Naumov, A.P. 1972. Tetraethylammonium ion inhibition of potassium conductance of the nodal membrane. Biochim. Biophys. Acta. 290:248-255

Pascual, J.M., Shieh, C.C., Kirsch, G.E., Brown, A.M. 1995. Multiple residues specify external tetraethylammonium blockade in voltage-gated potassium channels. Biophys. J. 69:428-434

Quinn, C.C., Begenisich, T. 2001. The influence of surface charges on quaternary ammonium block of Shaker $\mathrm{K}^{+}$channels. J. Membr. Biol. 182:233-243

Taglialatela, M., Vandongen, A.M., Drewe, J.A., Joho, R.H., Brown, A.M., Kirsch, G.E. 1991. Patterns of internal and external tetraethylammonium block in four homologous $\mathrm{K}^{+}$ channels. Mol. Pharmacol. 40:299-307

Tamargo, J., Caballero, R., Gomez, R., Valenzuela, C., Delpon, E. 2004. Pharmacology of cardiac potassium channels. Cardiovasc. Res. 62:9-33

Thompson, J., Begenisich, T. 2005. Two stable, conducting conformations of the selectivity filter in Shaker $\mathrm{K}^{+}$channels. J. Gen. Physiol. 125:619-629

Yost, C.S. 1999. Potassium channels: Basic aspects, functional roles, and medical significance. Anesthesiology 90:1186-1203

Zhou, Y., Morais-Cabral, J.H., Kaufman, A., MacKinnon, R. 2001. Chemistry of ion coordination and hydration revealed by a $\mathrm{K}^{+}$channel-Fab complex at $2.0 \AA$ A resolution. Nature 414:43-48 